\title{
Characterization of Regional Pulmonary Mechanics from Serial MRI Data
}

\author{
James Gee, Tessa Sundaram, Ichiro Hasegawa, \\ Hidemasa Uematsu, and Hiroto Hatabu \\ University of Pennsylvania, Departments of Bioengineering and Radiology \\ 3600 Market Street, Suite 370, Philadelphia, PA 19104, USA
}

\begin{abstract}
We describe a method for quantification of lung motion from the registration of successive images in serial MR acquisitions during normal respiration. MR quantification of pulmonary motion enables in vivo assessment of parenchymal mechanics within the lung in order to assist disease diagnosis or treatment monitoring. Specifically, we obtain estimates of pulmonary motion by summing the normalized cross-correlation over the lung images to identify corresponding locations between the images. The normalized correlation is robust to linear intensity distortions in the image acquisition, which may occur as a consequence of changes in average proton density resulting from changes in lung volume during the respiratory cycle. The estimated motions correspond to deformations of an elastic body and reflect to a first order approximation the true physical behavior of lung parenchyma. The method is validated on a serial MRI study of the lung, for which breath-hold images were acquired of a healthy volunteer at different phases of the respiratory cycle.
\end{abstract}

\section{Introduction}

The ability to quantify lung deformation is useful in characterizing the changes brought on by pulmonary pathology. Diseases such as idiopathic pulmonary fibrosis (IPF) and chronic obstructive pulmonary disease (COPD) change the structural properties of the lung parenchyma, and directly affect the lung's ability to normally expand and contract, [12]. COPD (or emphysema) increases lung compliance by promoting the destruction of the alveolar framework. Conversely, IPF stiffens the lung via an idiopathic increase in parenchymal connective tissue. It would be helpful to be able to observe and detect such morphologic changes and their effects on normal lung motion using medical imaging techniques.

Surrogate measures based on the motion of chest wall and diaphragm, 34, have been reported. However, magnetic resonance (MR) grid-tagging techniques allow the direct assessment of regional motion of moving structures in the human body, 567. The technique has recently been applied to evaluate local mechanical properties of the lung with highly promising results, 89. However, there are limitations due to (a) the relatively low spatial resolution of the estimated motion fields, stemming from the coarse grid intervals that are currently practicable, (b) fading of the grid by $\mathrm{T} 1$ decay time of the tissue, and (c) the 
difficulty of applying the method in 3-D. Moreover, because the T1 decay time of lung parenchyma is much shorter than the duration of the respiratory cycle, the technique can only be applied to study certain respiratory phases and not the entire breathing cycle, 8 .

In this work, a method is developed to determine a dense motion field-i.e., a displacement vector at every imaged point of the lung parenchyma - at each sampling time of a serial MR image sequence. The motion is estimated using the pulmonary vasculature and parenchymal structures as natural sources of spatial markers, without the requirement for explicit correspondence information, thus enabling the assessment and quantification of regional parenchymal deformation in the lungs over complete breathing cycles.

\section{Methods}

\subsection{Quantification of Lung Motion via Image Registration}

The goal of a registration algorithm is to find a spatial transformation or image warp that brings the features of one source image into alignment with those of a second, target image with similar content. Thus, registration algorithms can be used to determine the spatial locations of corresponding features in a sequence of MR pulmonary images. The computed correspondences immediately yield the displacement fields corresponding to the motion of the lung during the image sequence.

Many techniques for registration of MR images have been proposed, [10]. Simple methods assume that a rigid or affine transformation is sufficient to warp one image to another. Although such transformations may be sufficient to account for within-subject variation in rigid structures, to align features in lung images from different individuals or from the same individual acquired at different times, higher dimensional, non-rigid transformations are required in general.

A review of non-rigid registration techniques can be found in 11. In previous work, 12 13, we have developed a system in which an initial global affine registration of the source 3-D volume to the fixed target volume is performed to correct differences in object pose and location. This provides a starting point for an elastic registration algorithm, [12], which subsequently computes a local match, described by a dense displacement field $\mathbf{u}$ over the source image, that brings the detailed anatomy into register. The algorithm uses a finite element method iteratively to optimize an objective function $\pi$, which balances the pointwise similarity (or voxel-to-voxel correspondence) of the warped source and target images with the amount of deformation caused by $\mathbf{u}$ :

$$
\pi=\lambda \cdot \text { deformation }-\alpha \cdot \text { similarity },
$$

where $\lambda$ moderates the smoothness of the warps and $\alpha$ encodes the uncertainty in our image measurements, [12]. In this work, we obtain estimates of pulmonary 
motion by summing the normalized cross-correlation over the lung images to identify corresponding locations between the images, $I_{1}, I_{2},[10$ :

$$
\frac{\sum_{\mathbf{X}_{i} \in \mathcal{G}(\mathbf{X})} I_{1}\left(\mathbf{X}_{i}\right) I_{2}\left(\mathbf{X}_{i}\right)}{\left(\sum_{\mathbf{X}_{i} \in \mathcal{G}(\mathbf{X})} I_{1}\left(\mathbf{X}_{i}\right) I_{1}\left(\mathbf{X}_{i}\right) \sum_{\mathbf{X}_{i} \in \mathcal{G}(\mathbf{X})} I_{2}\left(\mathbf{X}_{i}\right) I_{2}\left(\mathbf{X}_{i}\right)\right)^{1 / 2}}
$$

where $\mathcal{G}(\mathbf{X})$ is a neighborhood of voxels around the location $\mathbf{X}$ at which the images are being compared. By measuring the similarity over a neighborhood of each voxel and thus taking into account the voxel's local image structure, the normalized correlation should yield better results than pointwise measures such as the squared intensity difference. Moreover, unlike the latter measure which assumes that the intensity of any point tracked during motion is constant, [14], the normalized correlation is robust to linear intensity distortions in the image acquisition, which may occur in our studies as a consequence of changes in average proton density resulting from changes in lung volume during the respiratory cycle.

For the deformation term in (11), we use the linear elastic strain energy, [15]:

$$
\frac{1}{2} \int_{V} \boldsymbol{\sigma}: \varepsilon d V
$$

where $\boldsymbol{\sigma}$ and $\varepsilon$ denote the stress and strain tensors, respectively 15. Their elastic constitutive relation is given by the generalized Hooke's law: in indicial notation, $\sigma_{i j}=D_{i j k l} \varepsilon_{k l}$, where the elastic coefficients $D_{i j k l}(\mathbf{X})$ reduce to two constants known as the Lamé parameters for the homogeneous, isotropic material idealization used in the current work. The estimated motions thus correspond to deformations of an elastic body and reflect to a first order approximation the true physical behavior of lung parenchyma - the source image volume takes on the properties of an idealized elastic material under the influence of loads which act along the gradients in the similarity between the image and the target volume.

\subsection{In Vivo Assessment of Regional Pulmonary Mechanics}

From the calculated displacement field, u, representing pulmonary motion, a quantitative description of the tissue deformation induced in the lung can be derived in the form of the (finite) Lagrangian strain tensor, $\varepsilon^{*},[15]: \varepsilon^{*}=$ $1 / 2\left\{\nabla \mathbf{u}+(\nabla \mathbf{u})^{\mathrm{T}}+(\nabla \mathbf{u})^{\mathrm{T}} \cdot(\nabla \mathbf{u})\right\}$, where $\nabla \mathbf{u}$, the second-order displacement gradient, can be numerically computed, 13. When the displacement gradients are small, the product terms in $\varepsilon^{*}$ can be neglected to obtain the infinitesimal or small-deformation strain tensor, $\boldsymbol{\varepsilon}$, whose components with respect to rectangular Cartesian coordinates $\mathbf{X}$ (in the undeformed configuration) are given by $\varepsilon_{i j}=1 / 2\left(\partial u_{i} / \partial X_{j}+\partial u_{j} / \partial X_{i}\right)$.

Visualization and analysis of either strain tensor over the imaged anatomy can be accomplished by mapping pointwise various scalar indices derived from the tensors. Specifically, the principal strains, which include the maximum and 
minimum normal strains experienced at a point, are characterized by the tensor eigenvalues. Two frame-independent indices are the first and second moments of the distribution of tensor eigenvalues: the former represents the trace of the tensor or the sum of its eigenvalues and provides an overall measure of strain magnitude; and the second moment measures the variance of the eigenvalues, which indicates the degree of directional bias (or anisotropy) in the strain profile. The strain anisotropy can be further analyzed by examining the off-diagonal elements or shear strain components of $\varepsilon^{*}$ or $\varepsilon$. The strain magnitude and anisotropy measures depend only on the tensor eigenvalues and not on the eigenvectors. The eigenvectors, however, also reveal important information about the observed deformation, particularly in regions of the lung where the strain anisotropy is the greatest. The eigenvectors are the directions along which the principal strains are experienced; thus, they characterize the orientation of the strain profile.

\section{$3 \quad$ Experimental Results}

Figures 11 and 2 demonstrate preliminary results obtained in an MRI study of simulated lung motion, [16]. Images were acquired of a young healthy male volunteer on a 1.5 T whole body MRI scanner (Signa Horizon, General Electric) using a two-dimensional true FISP sequence, with $\mathrm{TR}=3.0 \mathrm{msec}$, $\mathrm{TE}=1.5$ msec and matrix size $=128 \times 128$, resulting in a total acquisition time of $0.4 \mathrm{sec}$ per image. Other imaging parameters were as follows: flip angle $=35$ degrees; field of view $=450 \mathrm{~mm}$; and section thickness $=10 \mathrm{~mm}$. Five sagittal images of the right lung were obtained with breath-holding at different phases of the respiratory cycle, spanning full inhalation (phase I) to full exhalation (phase V).

Each image was non-rigidly registered to the next image in the sequence. In Fig. 1, the registration transformation between the first two images in the sequence, represented as a field of displacement vectors, is shown superimposed on the first image, where the vectors indicate the corresponding locations in the second image and thus provide a direct, quantitative measure of the lung motion between the images. The associated finite strain tensor was computed over each displacement field and is visualized in Fig. 2 using an ellipse to depict each tensor, where the major and minor axes of the ellipse are directed along the tensor eigenvectors, respectively, and scaled by the corresponding principal strain values.

The dynamics of respiratory lung deformation was studied by examining the measurements over the image sequence, as exemplified by the series of motion and strain tensor maps depicted in Fig. 2. The diaphragm rises as the lung volume decreases from total lung capacity (TLC) to residual volume (RV). At the same time, the posterior portion of the lung displaces superiorly, whereas the anterior portion is displaced posteriorly and superiorly. Regional parenchymal strain appears oriented toward the pulmonary hilum, with strain magnitude maximal at the mid-cycle of the expiratory phase.

These preliminary data - specifically, the estimated lung motion between successive images - have been validated, [17]. The validation protocol was designed 


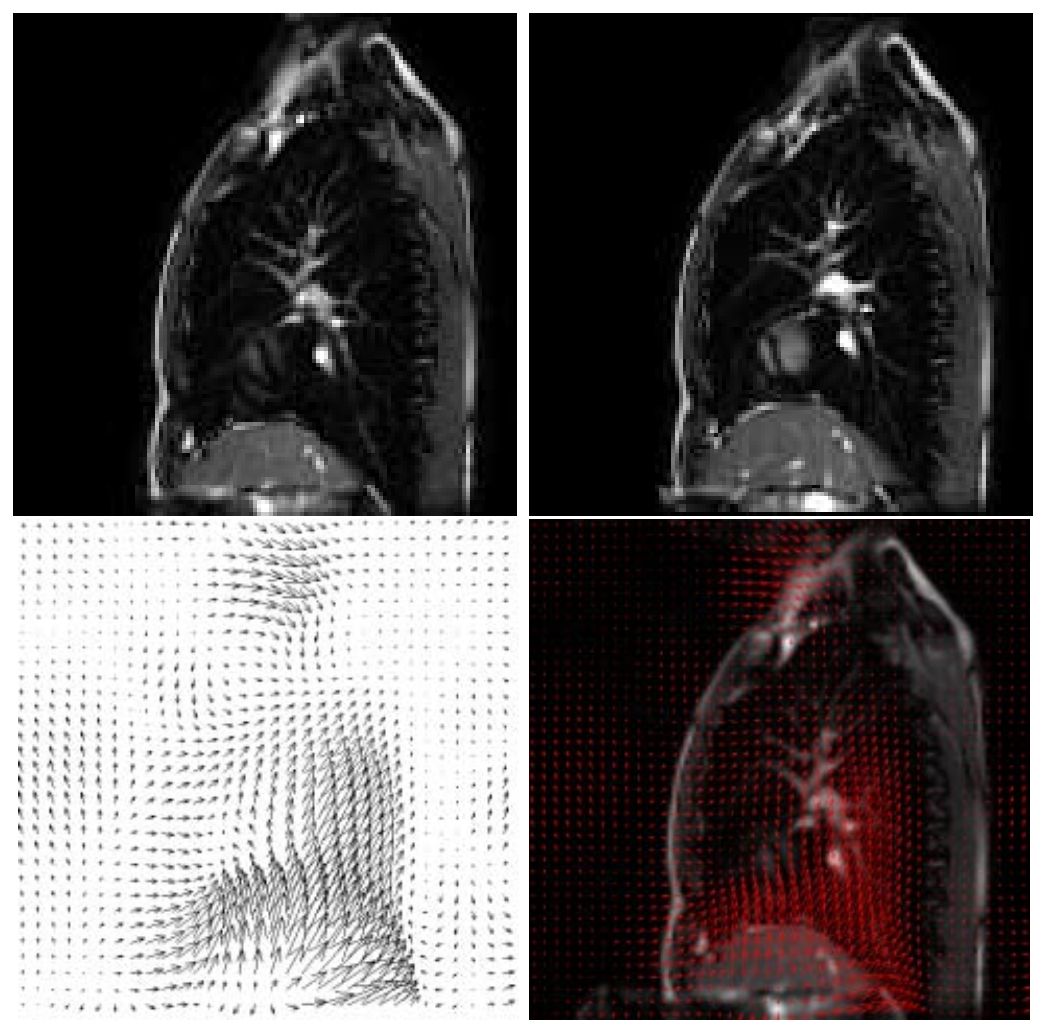

Fig. 1. Illustration of the estimated lung motion between two consecutive frames (top row) in a sequence of MR images. The registration transformation between the images, represented as a field of displacement vectors (bottom left), is shown superimposed on the first image (bottom right), where the vectors indicate the corresponding locations in the second image and thus provide a direct, quantitative measure of the lung motion between the images.

around the identification and tracking of 22 landmarks chosen at the bifurcations of pulmonary vasculature. Experts skilled at reading MR images independently identified the landmarks at each of the five phases. Three sets of expert data were then averaged to form a "ground truth" set of landmarks for the expiratory phase. The displacements of these truth landmarks were calculated and compared to the displacements determined by the registration algorithm at the landmark locations (Fig. 31). No significant difference was detected between the three sets of expert landmarks and the truth landmark set $(p>0.9)$, suggesting a minor contribution by user variation to the overall error. In addition, a significant difference was neither observed between the magnitudes $(p>0.3)$ nor the directions $(p>0.4)$ of the registration-derived and landmark-derived displacements. The average error between the endpoints of the displacement vectors was $1.14 \pm 0.93$ pixels. 


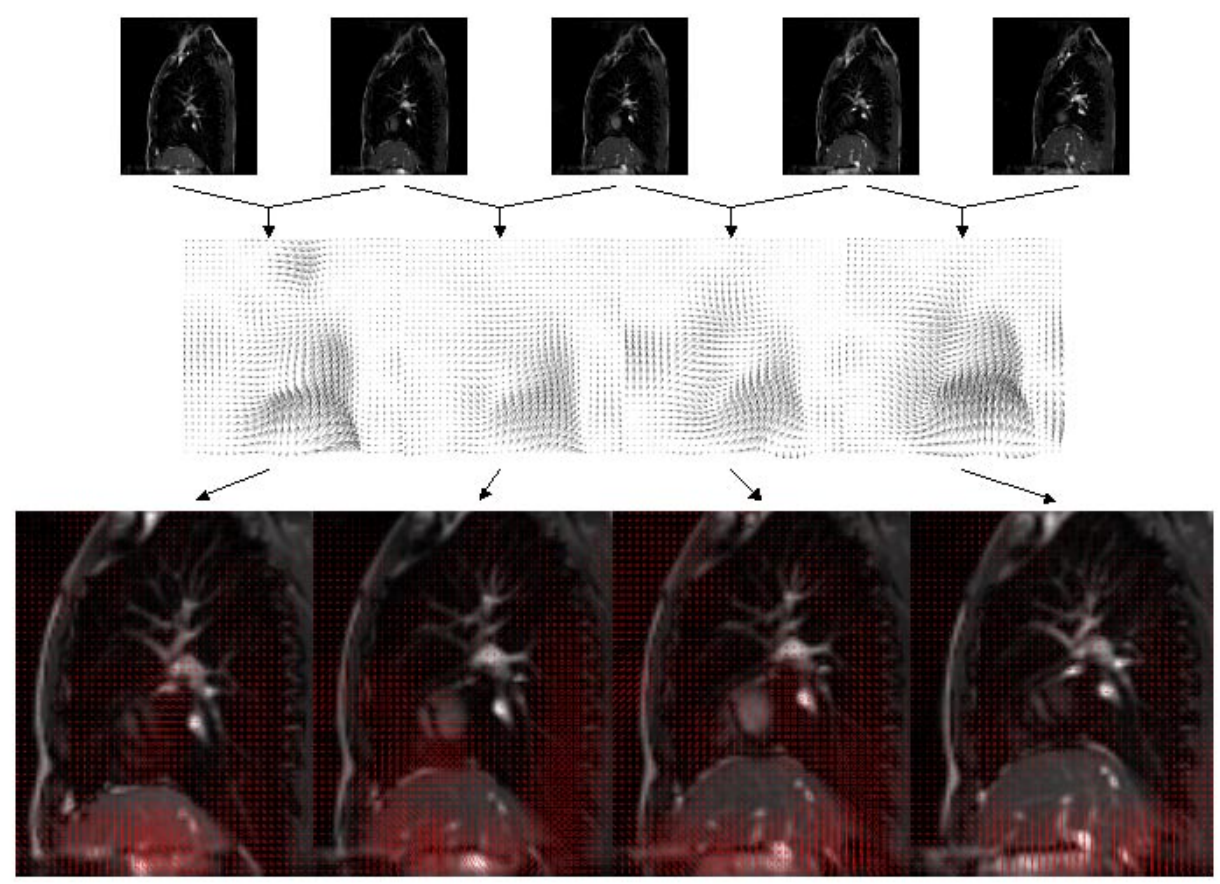

Fig. 2. Visualization of the dynamics of respiratory lung deformation. The calculated motion fields (middle row) between successive pairs of MR images (top row) over the expiratory phase of the respiratory cycle are shown along with the induced strain tensor fields (bottom row), where each tensor is depicted using an ellipse with major and minor axes directed along its eigenvectors, respectively, and scaled by the corresponding principal strain values.

\section{Discussion}

The recent development of fast MR imaging techniques has made possible detailed, non-invasive imaging of pulmonary parenchyma by overcoming the inherent difficulties associated with lung imaging, including low proton density, severe magnetic field susceptibility, and respiratory and cardiac motion artifacts. A gradient-echo sequence with ultra-short TE and a single-shot fast SE sequence can together provide a platform for MR imaging of the lung. Pulmonary perfusion can be assessed using a T1-weighted gradient-echo sequence with ultra-short TE and contrast agents. Regional pulmonary ventilation can also be evaluated by MR using molecular oxygen or laser-polarized Xe-129 and He-3. In the current work, we explore the use of fast MR imaging to study lung mechanics, which should provide a third, key diagnostic dimension in the assessment of pulmonary function, augmenting the information provided by studies of ventilation and perfusion. Specifically, the determination of regional biomechanical parameters in the lung has the potential to assist detection of early and/or localized pathological processes. Moreover, quantitative measurements of these 

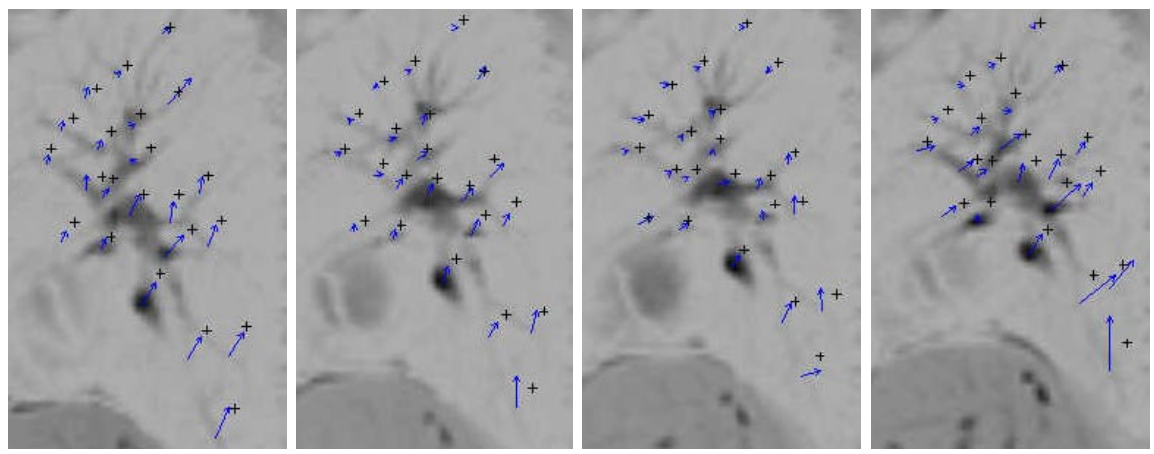

Fig. 3. Enlarged comparison of the landmark-based (arrows) and registration-derived (plus signs) displacements for each of the four motion fields. Only the endpoints of the registration-derived vectors are shown.

physical properties could provide an objective evaluation of therapeutic effects in various pulmonary disorders

The feasibility of a novel method is demonstrated here for quantifying lung motion (and, in turn, in vivo assessment of parenchymal deformation) from a sequence of pulmonary MR images acquired over the respiratory cycle. The method determines the lung motion between sequential frames by estimating the spatial transformation which brings the images into register. The approach has been verified over simulated motion data, but it remains to be tested on image sequences acquired without breath-holding, which is planned for the future.

\section{References}

1. Pratt, P. C.: Emphysema and Chronic Airways Disease. In: Dail, D., Hammar, S. (eds.): Pulmonary Pathology. Springer-Verlag, New York (1988) 654-659

2. Fulmer, J. D., Roberts, W. C., von Gal, E. R., Crystal, R. G.: MorphologicPhysiologic Correlates of the Severity of Fibrosis and Degree of Cellularity in Idiopathic Pulmonary Fibrosis. J. Clin. Invest. 63 (1979) 665-676

3. Suga, K., Tsukuda, T., Awaya, H., Takano, K., Koike, S., Matsunaga, N., Sugi, K., Esato, K.: Impaired Respiratory Mechanics in Pulmonary Emphysema: Evaluation With Dynamic Breathing MRI. J. Magn. Reson. Imaging 10 (1999) 510-520

4. Cluzel, P., Similowski, T., Chartrand-Lefebvre, C., Zelter, M., Derenne, J. P., Grenier, P. A.: Diaphragm and Chest Wall: Assessment of the Inspiratory Pump With MR Imaging-Preliminary Observations. Radiology 215 (2000) 574-583

5. Zerhouni, E. A., Parish, D. M., Rogers, W. J., Yang, A., Shapiro, E. P.: Human Heart Tagging With MR Imaging-A Method for Noninvasive Assessment of Myocardial Motion. Radiology 169 (1988) 59-63

6. Axel, L., Dougherty, L.: MR Imaging of Motion with Spatial Modulation of Magnetization. Radiology 171 (1989) 841-845

7. Axel, L., Dougherty, L.: Heart Wall Motion: Improved Method of Spatial Modulation of Magnetization for MR Imaging. Radiology 172 (1989) 349-350 
8. Chen, Q., Mai, V. M., Bankier, A. A., Napadow, V. J., Gilbert, R. J., Edelman, R. R.: Ultrafast MR Grid-Tagging Sequence for Assessment of Local Mechanical Properties of the Lungs. Magn. Reson. Med. 45 (2001) 24-28

9. Napadow, V. J., Mai, V., Bankier, A., Gilbert, R. J., Edelman, R., Chen, Q.: Determination of Regional Pulmonary Parenchymal Strain During Normal Respiration Using Spin Inversion Tagged Magnetization MRI. J. Magn. Reson. Imag. 13 (2001) 467-474

10. Maintz, J. B. A., Viergever, M. A.: A Survey of Medical Image Registration. Medical Image Analysis 2 (1998) 1-31

11. Lester, H., Arridge, S. R.: A Survey of Hierarchical Non-Linear Medical Image Registration. Pattern Recognition 32 (1999) 129-149

12. Gee, J. C., Bajcsy, R. K.: Elastic Matching: Continuum Mechanical and Probabilistic Analysis. In: Toga, A. W. (ed.): Brain Warping. Academic Press, San Diego (1999) 183-197

13. Gee, J. C., Haynor, D. R.: Numerical Methods for High-Dimensional Warps. In: Toga, A. W. (ed.): Brain Warping. Academic Press, San Diego (1999) 101-113

14. Horn, B., Schunck, B.: Determining Optical Flow. Artif. Intell. 17 (1981) 185-203

15. Malvern, L. E.: Introduction to the Mechanics of a Continuous Medium. PrenticeHall, Englewood Cliffs (1969)

16. Hatabu, H., Ohno, Y. Uematsu, H., Nakatsu, M., Song, H. K., Oshio, K., Gefter, W. B., Gee, J. C.: Lung Biomechanics Via Non-Rigid Registration of Serial MR Images. Proc. ISMRM 9th Scientific Meeting (2001) 2008

17. Sundaram, T. A., Gee, J.C., Hasegawa, I., Uematsu, H., Hatabu H.: Validation of A Registration Algorithm for Computing Lung Deformation From MR Images. Proc. ISMRM 10th Scientific Meeting (2002) in press 